\title{
Efeito agudo do pré-condicionamento isquêmico sobre o desempenho intermitente de basquetebolistas de elite
}

Acute effect of ischemic preconditioning on the intermittent performance of elite basketball players

\author{
Rodney Coelho da Paixão, \\ Gustavo Ribeiro da Mota ${ }^{1^{*}}$ \\ Edicarlos Machado Siqueira ${ }^{3}$ \\ Moacir Marocolo ${ }^{4}$
}

\section{Resumo:}

Objetivo: Verificar o efeito agudo do pré-condicionamento isquêmico ( $\mathrm{PCl}$ ) sobre a capacidade de realizar esforços intensos de alta demanda aeróbia em basquetebolistas de elite. Métodos: Onze atletas profissionais de basquete (25,4 $\pm 6,9$ anos; $200 \pm 10$-cm estatura; $97,7 \pm 11,2-\mathrm{kg}$ de massa corporal) realizaram de maneira cruzada, randomizada e balanceada o teste YoYolntermittent Recovery Test Level 1 (YoYolR1) logo após PCl (4 ciclos de 5-min de isquemia - pressão de $250 \mathrm{mmHg}$ e 5 -min de reperfusão - sem pressão externa) ou SHAM (placebo-controle, mesmo procedimento do $\mathrm{PCl}$, porém com pressão de $20 \mathrm{mmHg}$ na fase de "oclusão"). Resultados: Não houve diferença significativa ( $\mathrm{p}=$ $0,21)$ entre as condições PCI vs. SHAM ( $P C l=35 \pm 7,8$ estágios do YoYolR1 vs. SHAM $=37,5 \pm 8,2)$. Conclusão: Agudamente o PCl não promove melhora na capacidade de executar esforços intermitentes-intensos e com alta demanda aeróbia entre jogadores profissionais de elite de basquete.

Palavras-chave: ergogênico, intensidade, basquetebol.

\begin{abstract}
:
Objective: To evaluate the acute effect of ischemic preconditioning (IPC) on the ability to perform a highintense aerobic exercise in elite basketball players. Methods: Eleven professional basketball athletes (25.4 \pm 6.9 years, $200 \pm 10-\mathrm{cm}$ height, $97.7 \pm 11.2 \mathrm{~kg}$ of body mass) performed the YoYo Intermittent Recovery test Test Level 1 (YoYolR1) in a crossover, randomized and balanced design immediately after IPC (4 cycles of 5-min ischemia - $250 \mathrm{mmHg}$ pressure and 5-min reperfusion - no external pressure) or SHAM (placebo-control, same IPC procedure, but with $20 \mathrm{mmHg}$ in the "occlusion" phase). Results: There was no significant difference $(p=0.21)$ between IPCI vs. SHAM (IPC $=35 \pm 7.8$ stages of YoYolR1 vs. SHAM $=37.5 \pm 8.2$. Conclusion: Acute IPC does not promote improved ability to perform a
\end{abstract} high-intense aerobic exercise in elite basketball players.

Keywords: ergogenic, intensity, basketball.

Afiliação dos autores

Grupo de pesquisa em Desempenho Humano e Esporte, Departamento de Ciências do Esporte, Instituto de Ciências da Saúde, Universidade Federal do Triângulo Mineiro, Uberaba, Minas Gerais, Brasil

${ }^{2}$ Programa de Pós-Graduação em Genética e Bioquímica, Instituto de Biotecnologia, Universidade Federa de Uberlândia, Uberlândia, Minas

Gerais, Brasil
${ }^{3}$ Faculdade de Educação Física e Fisioterapia, Universidade Federal de Uberlândia, Uberlândia, Minas Gerais, Brasil

${ }^{4}$ Grupo de pesquisa em Fisiologia e Desempenho Humano,

Departamento de Fisiologia,

Universidade Federal de Juiz de

Fora, Juiz de Fora, Minas Gerais, Brasil.

\section{${ }^{*}$ Autor correspondente}

Grupo de pesquisa em Desempenho Humano e Esporte, Departamento de Ciências do Esporte, Instituto de Ciências da Saúde, Universidade Federal do Triângulo Mineiro, Av.

Tutunas, 490, CEP 38061-500,

Uberaba, Minas Gerais, Brasil. e-mail: gustavo.mota@uftm.edu.br

Conflito de interesses

Os autores declararam não haver conflito de interesses.

Processo de arbitragem

Recebido: 19/06/2018 Aprovado: 09/07/2018 


\section{Introdução}

O pré-condicionamento isquêmico $(\mathrm{PCl})$ alterna oclusão e liberação do fluxo sanguíneo muscular, por meio de torniquetes alocados nos membros inferiores ou superiores (parte proximal), antes da realização do exercício físico. O PCl tem sido testado como estratégia para melhorar o desempenho humano em diferentes tipos de atividades ${ }^{1-6}$, embora resultados conflitantes existam $^{7,8}$.

Diante de alguns achados positivos, alguns autores inclusive compararam o efeito do $\mathrm{PCl}$ com semanas de treinamento em altitude $^{9}$, e outros defendem que o $\mathrm{PCl}$ pode ser apontado como tipo de "doping natural"10. Aos encorajadores resultados encontrados em estudos prévios, somam-se a relativa simplicidade e baixo custo necessários para a realização da manobra, o que garante sua acessibilidade e aplicabilidade no ambiente esportivo.

Crisafulli et al. ${ }^{10}$ verificaram que o $\mathrm{PCl}$ melhorou a potência muscular máxima de sujeitos fisicamente ativos em teste incremental máximo em cicloergômetro. Adicionalmente, trabalhando com o mesmo tipo de teste, estudo demonstrou aumento de 1,6\% para potência máxima e de 3\% para consumo máximo de oxigênio entre ciclistas treinados ${ }^{9}$. Adicionalmente, nadadores de elite completaram um sprint de 100-m com tempo de 0,7-s mais rápido, quando o teste foi precedido pelo $\mathrm{PCl}^{11}$. Apesar de aparentemente ser pequeno o efeito benéfico (isto é, apenas 0,7-s), como a amostra era de atletas de elite, tal diferença pode ser importante do ponto de vista prático.

$\mathrm{O}$ uso do $\mathrm{PCI}$ tem sido especialmente indicado e utilizado no contexto esportivo, já que entre atletas, preparadores físicos e técnicos há busca contínua para melhorar o desempenho. Mais especificamente em nível profissional, detalhes podem diferenciar o sucesso e a falha, o que torna o componente físico extremamente relevante ${ }^{12}$.

Curiosamente, revisões sistemáticas mostram que a maioria dos estudos sobre "PCl e Exercício Físico" analisou os efeitos dessa manobra em modalidades individuais como natação, corrida e ciclismo $0^{6,7}$. Apenas um estudo testou o efeito do $\mathrm{PCl}$ em atletas com histórico de participação em esportes coletivos ${ }^{13}$, outro em sprints repetidos ${ }^{5}$ e recentemente (um terceiro trabalho) testou exercício intermitente em futebolistas, porém, amadores $^{14}$. Logicamente, as respostas ao $\mathrm{PCl}$ em amadores podem ser diferentes daquelas encontradas em atletas profissionais ou de superior nível competitivo ${ }^{15}$

De fato, nenhum trabalho analisou o impacto do $\mathrm{PCl}$ em atletas de elite e durante atividades intermitentes semelhantes aquelas realizadas especificamente (e validadas) para 0 basquetebol ${ }^{16}$. Fundamental destacar que o princípio da especificidade deve (ou deveria) ser atendido em pesquisas envolvendo ciências do esporte, especialmente as que analisam novas estratégias ergogênicas ${ }^{17-20}$, para garantir sua aplicação prática.

Portanto, o objetivo do presente estudo foi verificar o efeito agudo do $\mathrm{PCl}$ sobre a capacidade de conduzir esforços intensos com alta demanda aeróbia entre atletas profissionais de elite de basquetebol. Nossa hipótese foi que o $\mathrm{PCl}$ poderia melhorar o desempenho no "YoYolntermittent Recovery Test Level1" test (YoYolR1), porque recente revisão sistemática sugere que exercícios com maior componente aeróbio possam ser mais influenciados pelo $\mathrm{PCl}^{6}$.

\section{Métodos}

\section{Sujeitos}

Onze jogadores profissionais de basquete vinculados a uma equipe da principal liga nacional (Novo Basquete Brasil - NBB) participaram como voluntários (Tabela 1). Na época da coleta de dados os atletas estavam em fase competitiva (campeonato estadual) e, simultaneamente, em período preparatório para o principal campeonato da temporada. Todos os sujeitos assinaram termo de Consentimento Livre e Esclarecido. O estudo foi aprovado pelo Comitê local de Ética e Pesquisa em Seres Humanos (2296/2012).
Tabela 1.

Características gerais da amostra (média \pm desvio-padrão).

$\begin{array}{ll}\text { Idade (anos) } & 25,4 \pm 6,9 \\ \text { Massa Corporal (kg) } & 97,7 \pm 11,2 \\ \text { Estatura (m) } & 2,0 \pm 0,1 \\ \text { Gordura Corporal (\%) } & 10,9 \pm 3,5 \\ \text { Volume Semanal de Treinamento (horas) } & 25,6 \pm 2,5\end{array}$

\section{Desenho Experimental}

O desenho experimental deste estudo seguiu um modelo crossover. A ordem na qual os protocolos $[\mathrm{PCl}$ ou Controle $(\mathrm{CON})$ ] foram executados foi randomicamente definida. Todos os jogadores tinham experiência com o YoYolR1, já que este teste era adotado pela comissão técnica para avaliações regulares. Portanto, não houve necessidade de realizarmos nenhuma familiarização. Adicionalmente, o pesquisador que aplicou o YoYolR1 estava cego para qual condição o atleta havia realizado previamente (se PCI ou SHAM controle) e todos os atletas não recebiam nenhuma informação sobre 0 desempenho no teste até o final do pesquisa (áudio do YoYolR1 em idioma desconhecido). O consumo de produtos com cafeína, chás e álcool foi proibido, bem como exercício vigoroso nas 48-h anteriores aos testes, conforme recomendado em outros trabalhos semelhantes da área ${ }^{21,22}$.

\section{Pré-condicionamento Isquêmico}

Durante a manobra $\mathrm{PCl}$ os sujeitos permaneceram sentados em uma cadeira. Um torniquete pneumático (28-100 MC-ITS $\left.{ }^{\Theta}\right)$ foi colocado em cada coxa e as fases de isquemia e reperfusão foram alternadas entre os membros. O PCl durou 40-min, com quatro ciclos de 5 -min de isquemia (pressão externa de 250 $\mathrm{mmHg}$ ) e 5-min de reperfusão (sem pressão externa). Para o protocolo SHAM ou controle uma pressão externa insuficiente para promover isquemia $(20 \mathrm{mmHg})$ foi adotada, como sugerido em estudos prévios, ${ }^{1,-11,23}$. O tempo ideal entre o final da aplicação do $\mathrm{PCl}$ e início do teste de desempenho ainda é incerto, variando entre 5 a $90-\min ^{7}$ e resultados benéficos foram encontrados dentro de $45-\min ^{1,} 5$. Assim, neste estudo, o aquecimento para o teste YoYolR1 iniciou-se com 5-min após o encerramento do $\mathrm{PCl}$. Objetivando evitar potencial efeito placebo ${ }^{24}$ ou nocebo relacionados com $\mathrm{PCl}$ e desempenho em exercícios ${ }^{14}$, no atual estudo informamos os atletas que ambas as manobras $(\mathrm{PCl} e$ controle) poderiam melhorar 0 desempenho. E, ainda, que nenhuma delas, apesar de eventual desconforto associado ao procedimento, poderia piorar 0 desempenho ou causar algo negativo à saúde ${ }^{14}$.

\section{YoYo Intermittent Recovery Test Level 1}

O YoYolR1 foi empregado para avaliar a capacidade dos atletas em realizar esforcos repetitivos e com alta demanda aeróbia $^{25}$. A escolha do YoYolR1 para o atual estudo ocorreu por ser teste válido e específico para o basquetebol, indicado para avaliar a aptidão aeróbia e a endurance relacionada ao jogo de basquetebol ${ }^{16}$. Resumidamente, em área previamente marcada (Figura 1), os sujeitos executaram corridas de $2 \times 20-\mathrm{m}$ com 10-s de intervalo com recuperação ativa. $O$ aquecimento consistiu na realização dos três primeiros estágios do YoYolR1. Os atletas esperavam 3-min passivamente para iniciar o teste que foi mantido até a exaustão. $O$ resultado foi considerado como a distância percorrida ${ }^{26}$.

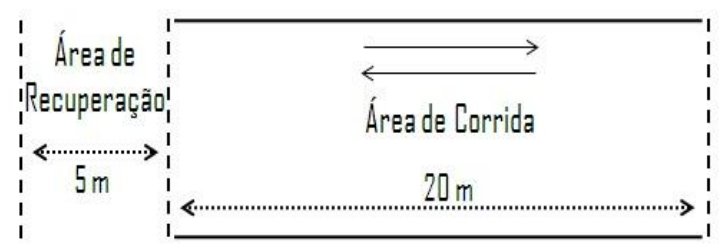

Figura 1. Esquema representativo do YoYolntermittent Recovery Test Level1.

\section{Análise Estatística}


O teste de Shapiro Wilk foi empregado para testar a normalidade dos dados e o teste $t$ pareado foi utilizado para comparar o desempenho entre os protocolos ( $\mathrm{PCl}$ vs. $\mathrm{CON}$ ). $\mathrm{O}$ nível de significância adotado foi de 5\%

\section{Resultados}

A distância percorrida no YoYolR1 não foi diferente $(p=$ 0,21 ) entre PCl e SHAM (controle - CTRL) (Figura 2). Considerando 0 número de estágios, $35.0 \pm 7.8$ foram completados no $\mathrm{PCl}$ e $37.5 \pm 8.2$ no CON. A Figura 3 mostra que a maioria dos atletas percorreu uma menor distância quando o teste foi precedido pela manobra $\mathrm{PCl}$.

A comparação de desempenho entre o primeiro e segundo testes revelou não haver diferenças significativas $(p=0,089)$.

\section{YoYo IR1}

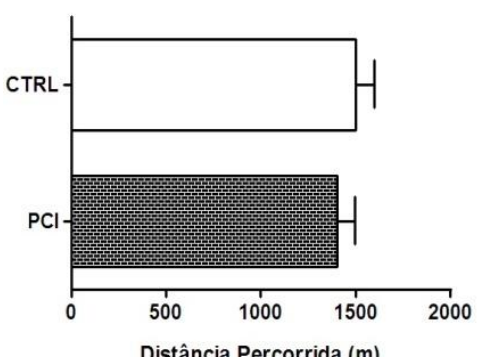

Figura 2. Comparação da distância percorrida no YoYolR1. PCI = précondicionamento isquêmico; $\mathrm{CTRL}=$ controle/SHAM; $\mathrm{N}$ =

YoYo IR1

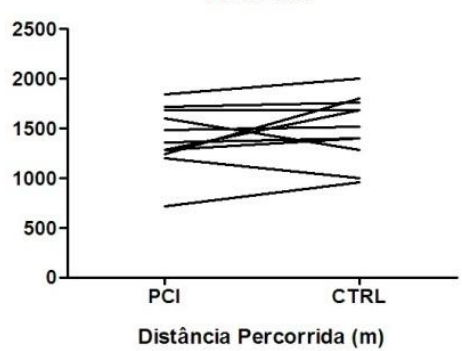

Figura 3. Desempenho individual na distância $(\mathrm{m})$ percorrida durante 0 YoYolR1 $. \mathrm{PCI}=$ pré-condicionamento isquêmico; $\mathrm{CTRL}=$ controle/SHAM; $\mathrm{N}=11$.

\section{Discussão}

$\mathrm{O} \mathrm{PCI}$ tem recebido recentemente proeminência na literatura científica $^{1}, 6,7,10,11,23$ e, de fato, trata-se de procedimento de baixo custo, fácil aplicação e sem efeitos colaterais adversos ${ }^{27}$. Entretanto, em nosso conhecimento, não havia informações concernentes do uso de $\mathrm{PCl}$ em modalidades coletivas, especialmente com atletas de elite fazendo parte da amostra. Assim, ao demonstrarmos originalmente que a manobra de $\mathrm{PCl}$ não garante melhor desempenho no YoYolR1 entre jogadores profissionais de basquete, expandimos o conhecimento sobre 0 relação $\mathrm{PCl}$-Exercício Físico e contribuímos efetivamente para o avanço do conhecimento científico.

Este estudo foi planejado com a proposta de unir um teste (YoYolR1) e um procedimento (PCl) relativo ao desempenho esportivo. No caso do YoYolR1, Krustrup et al. ${ }^{12}$ encontraram uma correlação significativa entre a distância percorrida e as corridas em alta intensidade durante um jogo de futebol professional $(r=0,76, p<, 05)$. Os autores pontuaram que 0 YoYolR1 poderia ser usado como indicador de desempenho em partidas oficiais de futebol feminino. Adicionalmente, o YoYolR1 foi testado e validado especificamente para o basquetebol, sendo indicado para avaliar a aptidão aeróbia e a endurance relacionada ao jogo de basquetebol ${ }^{16}$.

Sobre o efeito observado para $\circ \mathrm{PCl}$, o resultado encontrado no atual estudo não está em acordo com dados recentes que demonstraram benefícios para o desempenho atlético humano $1,10,11$. Ao adotarem o PCl entre nadadores altamente treinados, Jean-St-Michel et al. ${ }^{11}$ verificaram que os sujeitos foram mais rápidos em um sprint de 100-m. Em outro estudo, homens moderadamente treinados melhoraram em 34-s
- desempenho no teste de 5-km quando o exercício foi precedido pelo $\mathrm{PCl}(\mathrm{p}=0.027)^{1}$

De fato, a ineficiência da manobra no desempenho do YoYolR1 é contrária à nossa hipótese. Considerando a associação entre $\mathrm{PCl}$ e metabolismo aeróbio, especialmente sobre um possível efeito vasodilatador e maior oxigenação ofertada durante o exercício para músculos ativos ${ }^{9}$, nós acreditávamos que os atletas apresentariam melhores resultados após a manobra de PCI. De Groot et al. ${ }^{9}$ utilizaram a manobra de $\mathrm{PCl}$ em grupo de ciclistas treinados e envolvidos em um teste incremental máximo em cicloergômetro, demonstrando que além de maior potência pico, houve aumento no consumo máximo de oxigênio. Além disso, alguns aspectospositivos observados em modelos animais parecem ser relacionados com o exercício físico. $\mathrm{Li}$ e $\mathrm{He}^{28}$ verificaram que o efeito protetor do $\mathrm{PCl}$ sobre o miocárdio de coelhos $(60-\mathrm{min}$ de isquemia sustentada) estava relacionado à ativação de adenosina e com 0 aumento da densidade de receptores de adenosina. Radegran e Hellsten ${ }^{29}$ mostraram que a contração muscular influencia a atividade da AMP 5 'nucleotidase. E Saito et al. ${ }^{30}$ comprovaram que quando ratos adultos foram submetidos ao $\mathrm{PCI}$ houve maior oxigenação do músculo esquelético durante 0 exercício realizado.

Tendo em conta as informações contrárias disponíveis na literatura em comparação com nossos achados, os quais destacam os resultados positivos do $\mathrm{PCl}$ no desempenho atlético humano ${ }^{1,9-11}$, não podemos conceber uma explicação definitiva sobre o efeito imediato do $\mathrm{PCl}$ em modalidades esportivas coletivas, especialmente no basquete.

De qualquer modo, considerando que métodos e procedimentos deste estudo seguiram diretrizes similares àquelas adotadas em estudos prévios sobre $\mathrm{PCl}$ e exercício físico, acreditamos que aspectos externos devem ter contribuído para a falta de eficiência encontrada no protocolo $\mathrm{PCl}$. Primariamente, o aspecto motivacional pode ter modificado o desempenho no YoYolR1, já que estudos prévios confirmam a influência da condição psicológica no desempenho físico ${ }^{31}$. Ademais, considerando que a coleta de dados foi conduzida durante período que precedia a principal competição do ano, variações típicas da condição física ao longo da temporada podem ter determinado tais resultados ${ }^{32}$.

A amostra reduzida $(\mathrm{N}=11)$ pode ser considerada como potencial limitação do presente estudo. Entretanto, lembramos que equipes de basquetebol são geralmente compostas por pequeno número de atletas, quando comparadas com outras modalidades (ex., futebol, rúgbi) e, mais importante, o atual trabalho analisou o efeito do $\mathrm{PCl}$ exclusivamente em jogadores profissionais de elite, preenchendo lacuna crucial da literatura ${ }^{15}$. Finalmente, recomendamos cautela na extrapolação desses resultados para jogadores de outros níveis (ex., recreacionais/ amadores). Ressaltamos que a interpretação dos efeitos ergogênico do $\mathrm{PCl}$ sobre o desempenho humano considere futuros protocolos (ex., menos tempo total na manobra), com ajustes e inovações apropriadas para avançar o conhecimento específico ${ }^{15,}{ }^{33},{ }^{34}$ e ampliar o debate sobre esta temática relevante para a ciência do esporte.

\section{Conclusão}

A partir dos resultados encontrados no presente estudo, concluímos que agudamente o $\mathrm{PCl}$ não promove melhora na capacidade de executar esforços intermitentes-intensos e com alta demanda aeróbia entre basquetebolistas de elite. Sugere-se que estudos futuros adotem um período mais longo entre o tempo de execução da manobra $\mathrm{PCl}$ e o início do exercício, a fim de analisar o efeito de uma diferente "janela" de tempo.

\section{Referências}

1. Bailey TG, Jones H, Gregson W, Atkinson G, Cable NT, Thijssen DH. Effect of ischemic preconditioning on lactate accumulation and running performance. Med Sci Sports Exerc. 2012;44(11):2084-9.

2. Kjeld T, Rasmussen MR, Jattu T, Nielsen HB, Secher NH. Ischemic preconditioning of one forearm enhances static and dynamic apnea. Med Sci Sports Exerc. 2014;46(1):151-5.

3. Marocolo M, Marocolo IC, da Mota GR, Simao R, Maior AS, Coriolano HJ. Beneficial Effects of Ischemic Preconditioning in Resistance Exercise Fade Over Time. Int J Sports Med. 2016;37(10):819-24.

4. Marocolo M, Willardson JM, Marocolo IC, da Mota GR, Simao R, Maior AS Ischemic Preconditioning and Placebo Intervention Improves Resistance Exercise Performance. J Strength Cond Res. 2016;30(5):1462-9. 
5. Patterson SD, Bezodis NE, Glaister M, Pattison JR. The Effect of Ischemic Preconditioning on Repeated Sprint Cycling Performance. Med Sci Sports Exerc. 2015;47(8):1652-8.

6. Incognito AV, Burr JF, Millar PJ. The Effects of Ischemic Preconditioning on Human Exercise Performance. Sports Med. 2016;46(4):531-44.

7. Marocolo M, da Mota GR, Simim MA, Appell Coriolano HJ. Myths and Facts About the Effects of Ischemic Preconditioning on Performance. Int $J$ Sports Med. 2016;37(2):87-96

8. Paixao RC, da Mota GR, Marocolo M. Acute Effect of Ischemic Preconditioning is Detrimental to Anaerobic Performance in Cyclists. Int $\mathrm{J}$ Sports Med. 2014.

9. de Groot PC, Thijssen DH, Sanchez M, Ellenkamp R, Hopman MT. Ischemic preconditioning improves maximal performance in humans. Eur $\mathrm{J}$ Appl Physiol. 2010;108(1):141-6.

10. Crisafulli A, Tangianu F, Tocco F, Concu A, Mameli O, Mulliri G, et al. Ischemic preconditioning of the muscle improves maximal exercise performance but not maximal oxygen uptake in humans. J Appl Physiol. 2011:111(2):530-6.

11. Jean-St-Michel E, Manlhiot C, Li J, Tropak M, Michelsen MM, Schmidt MR, et al. Remote preconditioning improves maximal performance in highly trained athletes. Med Sci Sports Exerc. 2011;43(7):1280-6.

12. Krustrup $P$, Mohr M, Ellingsgaard $H$, Bangsbo J. Physical demands during an elite female soccer game: importance of training status. Med Sci Sports Exerc. 2005;37(7):1242-8.

13. Gibson N, Mahony B, Tracey C, Fawkner S, Murray A. Effect of ischemic preconditioning on repeated sprint ability in team sport athletes. J Sports Sci. 2015;33(11):1182-8.

14. Marocolo IC, da Mota GR, Londe AM, Patterson SD, Barbosa Neto O, Marocolo M. Acute ischemic preconditioning does not influence highintensity intermittent exercise performance. PeerJ. 2017;5:e4118.

15. Marocolo M, da Mota GR. Pré-condicionamento isquêmico e desempenho: há viabilidade/racionalidade na sua aplicação? Arq Cien Esp. 2018;6(1):2-

16. Castagna C, Impellizzeri FM, Rampinini E, D'Ottavio S, Manzi V. The YoYo intermittent recovery test in basketball players. J Sci Med Sport. 2008;11(2):202-8.

17. Mota GR, Magalhães CG, Azevedo PHSM, Ide BN, Lopes CR, Castardeli $\mathrm{E}$, et al. Lactate threshold in taekwondo through specifics tests. Journal of Exercise Physiology online. 2011;14(3):60-6.

18. da Silva BV, Simim MA, Marocolo M, Franchini E, da Mota GR. Optimal load for the peak power and maximal strength of the upper body in Brazilian Jiu-Jitsu athletes. Journal of Strength and Conditioning Research. 2015;29(6):1616-21.

19. Simim MAM, Silva BVC, Marocolo Júnior M, Mendes EL, Mello MTd, Mota GRd. Anthropometric profile and physical performance characteristic of the Brazilian amputee football (soccer) team. Motriz: Rev Educ Fis. 2013;19:641-8.
20. da Mota GR, Gomes LH, Castardeli E, Bertoncello D, José E, Vicente D, et al. Treinamento proprioceptivo e de força resistente previnem lesões no futebol. J Health Sci Inst 2010;28(2):191-3.

21. Meneghel AJ, Verlengia R, Crisp AH, Aoki MS, Nosaka K, da Mota GR, et al. Muscle damage of resistance-trained men after two bouts of eccentric bench press exercise. J Strength Cond Res. 2014;28(10):2961-6.

22. da Silva BVC, Marocolo M, Rogério FC, Dias IS, de Moura Simim MA, da Mota GR. Testes físicos discriminam praticantes de Brazilian Jiu-Jitsu? R Bras Ci e Mov. 2014;22(1):90-6.

23. Clevidence MW, Mowery RE, Kushnick MR. The effects of ischemic preconditioning on aerobic and anaerobic variables associated with submaximal cycling performance. Eur J Appl Physiol. 2012;112(10):364954.

24. Marocolo M, da Mota GR, Pelegrini V, Appell Coriolano HJ. Are the Beneficial Effects of Ischemic Preconditioning on Performance Partly a Placebo Effect? Int J Sports Med. 2015;36(10):822-5

25. Bangsbo J, laia FM, Krustrup P. The Yo-Yo intermittent recovery test : a useful tool for evaluation of physical performance in intermittent sports. Sports Med. 2008;38(1):37-51.

26. Krustrup P, Mohr M, Amstrup T, Rysgaard T, Johansen J, Steensberg A, et al. The yo-yo intermittent recovery test: physiological response, reliability, and validity. Med Sci Sports Exerc. 2003;35(4):697-705

27. Whetzel TP, Stevenson TR, Sharman RB, Carlsen RC. The effect of ischemic preconditioning on the recovery of skeletal muscle following tourniquet ischemia. Plast Reconstr Surg. 1997;100(7):1767-75.

28. Li YL, He RR. Protective effect of preconditioning on ischemic heart and characterization of adenosine receptors in ischemic rabbit hearts. Zhongguo Yao Li Xue Bao. 1995;16(6):505-8.

29. Radegran G, Hellsten $Y$. Adenosine and nitric oxide in exercise-induced human skeletal muscle vasodilatation. Acta Physiol Scand. 2000;168(4):575-91.

30. Saito $T$, Komiyama $T$, Aramoto $H$, Miyata $T$, Shigematsu $H$. Ischemic preconditioning improves oxygenation of exercising muscle in vivo. J Surg Res. 2004:120(1):111-8.

31. Sheldon KM, Zhaoyang R, Williams MJ. Psychological need-satisfaction, and basketball performance. Psychology of Sport and Exercise. 2013;14(5):675-81.

32. Koutedakis Y. Seasonal variation in fitness parameters in competitive athletes. Sports Med. 1995;19(6):373-92.

33. da Mota GR, Marocolo M. The Effects of Ischemic Preconditioning on Human Exercise Performance: A Counterpoint Sports Med. 2016;46(10):1575-6.

34. Marocolo M, Coriolano HA, Mourao CA, da Mota GR. Crucial Points for Analysis of Ischemic Preconditioning in Sports and Exercise. Med Sc Sports Exerc. 2017;49(7):1495-6. 